\title{
Candida albicans Osteochondromyelitis after Gastroesophageal Surgery: Two Case Reports
}

\author{
Abdulwares Meiwandi ${ }^{1}$ Hubert Zirngibl ${ }^{2}$ Ahmet Bozkurt ${ }^{1}$ \\ 1Department of Plastic, Reconstructive, Aesthetic and Hand \\ Surgery, Helios University Hospital Wuppertal, Wuppertal, \\ Germany \\ 2Division of Surgery II, Witten-Herdecke University, Wuppertal, \\ Germany \\ Indian J Plast Surg 2021;54:232-234.
}

\begin{abstract}
Address for correspondence Abdulwares Meiwandi, MD, Department of Plastic, Reconstructive, Aesthetic and Hand Surgery, Helios University Hospital Wuppertal, 42283 Wuppertal, Germany (e-mail: a.meiwandi@icloud.com).
\end{abstract}

\begin{abstract}
Keywords

- candida albicans

- osteomyelitis

- sternum

- ribs

- surgical wound infection

Candida osteochondromyelitis is a rare complication after thoracoabdominal surgery. We herein report two such cases after uncomplicated thoracoabdominal surgery, who presented with chronic postsurgical site infection and fistula. CT scans showed fistulas reaching the costochondral areas of the fifth rib. Inflammatory parameters were not elevated. Both patients were treated successfully after the initiation of systemic antimycotic treatment and surgical debridement. We conclude that $C$. albicans infections should always be considered in cases of chronic postoperative surgical site infections after thoracoabdominal surgery. Additional risk factors do not need to be present. Appropriate therapy consists of the application of systemic antimycotics and surgical debridement.
\end{abstract}

\section{Introduction}

Candida osteochondromyelitis of the sternum and ribs is a rarely reported condition that is most often reported after cardiac or thoracic surgery in individuals who exhibit additional risk factors such as immunosuppression, previous candidemia, parenteral nutrition, diabetes mellitus, drug abuse, prolonged stay at the ICU, and the use of broad-spectrum

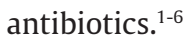

We herein report two rare cases of deep-seated Candida osteochondromyelitis of the ribs and sternum after gastroesophageal surgery. To our knowledge, this is only the seventh and eighth case published in the entire literature that reports Candidal chondro-osteomyelitis of the sternum and ribs after thoracoabdominal esophageal surgery. ${ }^{7-9}$ Late diagnosis is common, and subsequent antifungal treatment is often delayed.

\section{Case Presentation}

Case 1

A58-year-old male patientexhibited a chronic postsurgical site infection after uncomplicated thoracoabdominal esophageal

DOI https://doi.org/

10.1055/s-0041-1731854

ISSN 0970-0358 resection and sleeve gastrectomy for squamous carcinoma of the esophagus 16 months before consultation. A fistula presented itself 7 months postsurgery, measuring $5 \times 3 \mathrm{~cm}^{2}$ at the medial portion of the anterolateral thoracal incision at the level of the costochondral transition of the 5th rib.

Laboratory results showed no elevated inflammation parameters (procalcitonin [PCT], C-reactive protein [CRP] and leukocytes). Preoperative CT was taken to show the extent of the disease (see - Fig. 1 B). The fistula was excised in total, including the affected rib, and the defect was closed primarily. However, the fistula recurred after 5 months. We excised the 5th to 7th ribs and most parts of the sternum, as they were affected. Bony and chondral probes were taken. This time, mainly Candida albicans was detected. We initiated long-term antifungal therapy with intravenous (IV) caspofungin. Fifty mg was given for 3 weeks after loading dose of $70 \mathrm{mg}$. After multiple debridements and long-term vacuum-assisted closure (VAC) therapy, no C. albicans was detectable any longer in the taken probes. The wound was finally closed through a pedicled latissimus dorsi myocutaneous flap from the left side. The flap healed without any complications, and the patient was discharged 3 weeks after

(C) 2021. Association of Plastic Surgeons of India.

This is an open access article published by Thieme under the terms of the Creative Commons Attribution-NonDerivative-NonCommercial-License, permitting copying and reproduction so long as the original work is given appropriate credit. Contents may not be used for commercial purposes, or adapted, remixed, transformed or built upon. (https://creativecommons.org/licenses/by-nc-nd/4.0/).

Thieme Medical and Scientific Publishers Pvt. Ltd. A-12, 2nd Floor, Sector 2, Noida-201301 UP, India 


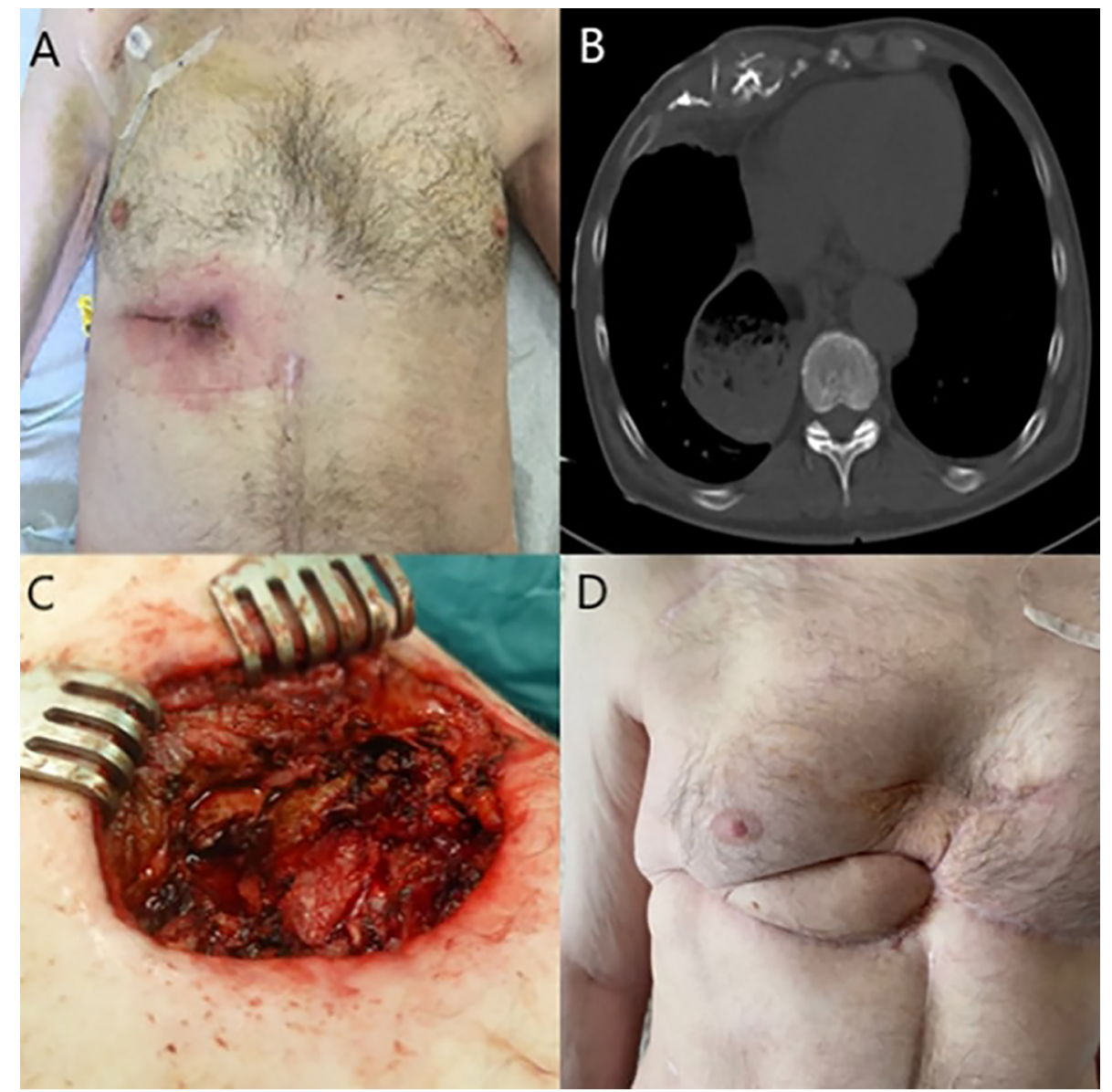

Fig. 1 Case 1 of Candida osteochondromyelitis. (A) A preoperative photograph of a purulent fistula overlying the fifth rib and surrounding candida infection of the skin at first admission. (B) Corresponding CT scan. (C) Intraoperative photograph. (D) Follow-up photograph 1-year postop.

wound closure. Oral fluconazole $400 \mathrm{mg}$ was given for additional 4 months. No fistula or any signs of postsurgical site infections were present at -1 year follow-up.

\section{Case 2}

An 81-year-old female presented with chronic fistula at the region of the right 5th and 6th ribs after anterolateral thoracotomy and sleeve gastrectomy of an upside down stomach 3 years ago. Laboratory parameters were normal. Preoperative CT thorax images showed a cutaneous fistula with thickening of the adjacent cartilage of the 5th rib and surrounding soft-tissue proliferation as a sign of an inflammatory process ( - Fig. 2B). During the first debridement, we excised the fistula superficially and took samples for microbiological and histological evaluation. These revealed the presence of $C$. albicans and, to a smaller degree, S. aureus. We then started an intensive local and systemic therapy with fluconazole $400 \mathrm{mg}$ after loading dose of $800 \mathrm{mg}$. After repeated wound revision and VAC therapy, the wound finally showed a clean condition, so that we were able to cover the resulting defect with a latissimus dorsi myocutaneous flap ( - Fig. 2C). The patient took fluconazole $400 \mathrm{mg}$ orally for another 4 months. In a CT control, approximately 2 months after surgery, there were no more signs of osteochondromyelitis. No further fistula has presented itself at 1-year follow-up.

\section{Discussion}

Candidal osteochondromyelitis after thoracoabdominal esophageal surgery is a rare condition that has previously only been described in a case series of four patients by Sica et al and in two other case reports by Eves et al and Nowicki et al.7-9 Slenker et al and Gamaletsou et al each have reviewed more than 200 cases in the literature of Candida osteomyelitis. ${ }^{1,3}$ Two-third of these infections occurred through hematogenous dissemination, and most of them have conditions with immunosuppression. In the case of Candida osteochondromyelitis related to gastroesophageal surgery, we believe that the fungal agents probably enter the costal cartilage after initial surgery and are disseminated from there onto the skin. $C$. albicans is a facultative pathogen that colonizes the esophageal mucosa. ${ }^{7-10}$ Patients generally present a few weeks/months after esophageal surgery with a surgical site infection at the thoracal incision. $^{7-9}$ Our first patient was on oral corticosteroid for severe rheumatoid arthritis, which is a proven risk factor. Both our cases presented with fistula, which did not respond to conservative therapy. CT or MRI scans with contrast agents are helpful to determine the extent of infection and visualize the area that is affected..$^{-9}$ After microbiological confirmation, these patients need long-term therapy with systemic antifungal agents such as fluconazole, based on sensitivity. ${ }^{1-8}$ The wound should be radically debrided, to clear the deep-seated 


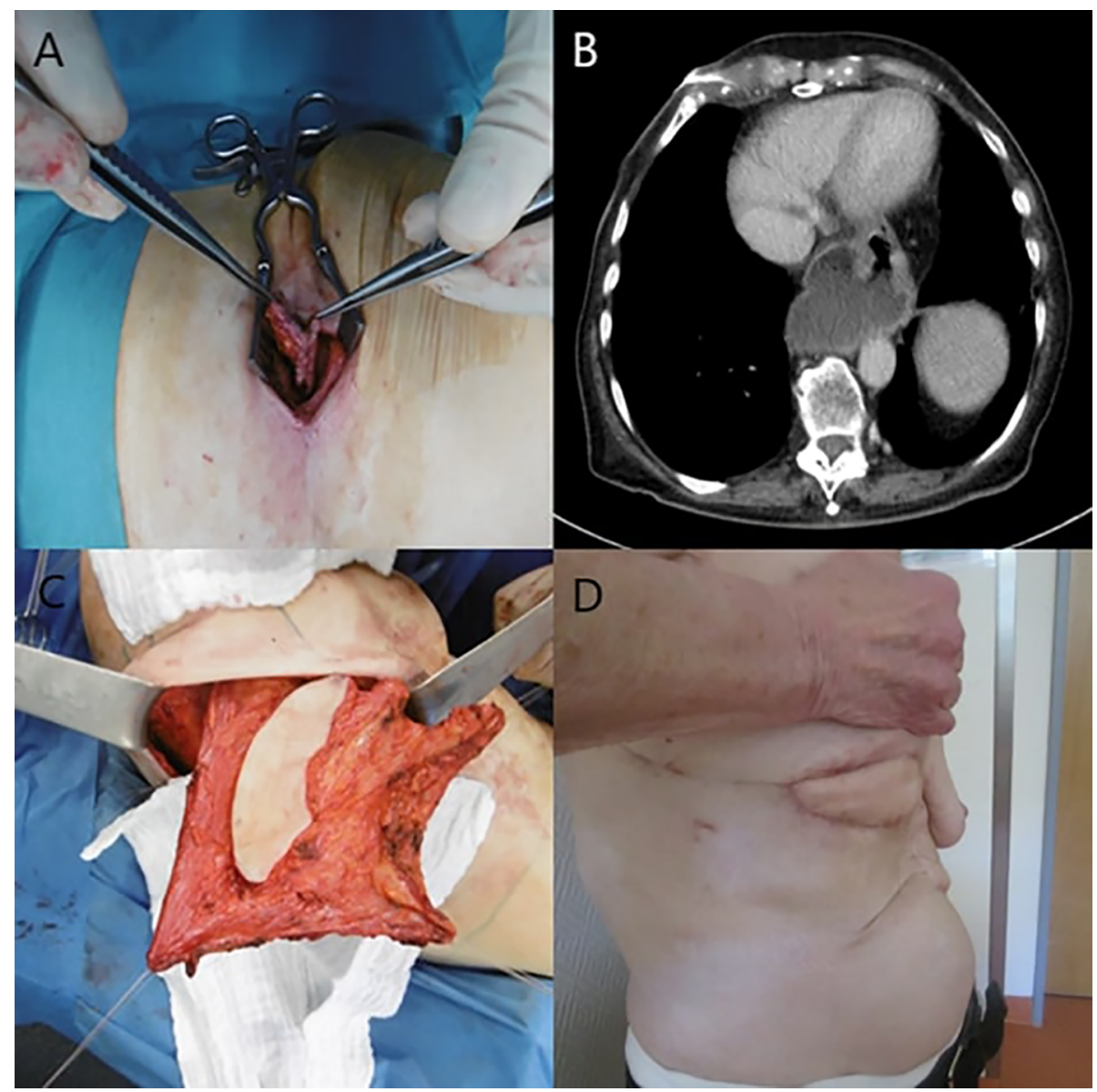

Fig. 2 Case 2 of Candida osteochondromyelitis. (A) Intraoperative photograph of the incised fistula down to the underlying fifth rib, visible Candida infection of the surrounding skin. (B) Corresponding CT scan. (C) Intraoperative photograph of the latissimus dorsi muscle flap. (D) Follow-up photograph 6 months postop.

infection, which often involves adjacent areas with mixed pathogens. The prognosis of the patients is good after initiation of the appropriate therapy. ${ }^{7-9}$ However, without systemic administration of antimycotics, relapse is seen in most cases, , 1,3,4,6

In summary, clinicians should always be suspicious of a Candida infection in a chronic persistent exuding fistula after gastroesophageal surgery. The therapy of choice should be surgical wound debridement and systemic antimycotic therapy.

\section{Declaration}

None.

\section{Ethics Approval and Consent for Publication}

The study was performed in accordance with the principles of the Declaration of Helsinki. Written informed consent was obtained of this case report and any accompanying images.

\section{Conflict of Interests}

The authors declare that they have no competing interests.

\section{References}

1 Slenker AK, Keith SW, Horn DL. Two hundred and eleven cases of Candida osteomyelitis: 17 case reports and a review of the literature. Diagn Microbiol Infect Dis 2012;73(1):89-93
2 Gathe JC, Jr, Harris RL, Garland B, Bradshaw MW, Williams TW, Jr. Candida osteomyelitis. Report of five cases and review of the literature. Am J Med 1987;82(5):927-937

3 Gamaletsou MN, Kontoyiannis DP, Sipsas NV, et al. Candida osteomyelitis: analysis of 207 pediatric and adult cases (1970-2011) Clin Infect Dis 2012;55(10):1338-1351

4 Arias F, Mata-Essayag S, Landaeta ME, et al. Candida albicans osteomyelitis: case report and literature review. Int J Infect Dis 2004;8(5):307-314

5 Malani PN, McNeil SA, Bradley SF, Kauffman CA. Candida albicans sternal wound infections: a chronic and recurrent complication of median sternotomy. Clin Infect Dis 2002;35(11):1316-1320

6 Modrau IS, Ejlertsen T, Rasmussen BS. Emerging role of Candida in deep sternal wound infection. Ann Thorac Surg 2009;88(6):1905-1909

7 Eves S, Sayeed R, Potter M. A case of rib fungal osteomyelitis. J Plast Reconstr Aesthet Surg 2014;67(3):e81-e83

8 Sica G, Meissner S, Dawas K, Maynard N. Candida osteo-chondromyelitis complicating thoraco-abdominal esophageal surgery. Surg Infect (Larchmt) 2007;8(4):479-482

9 Nowicki JL, Dean NR, Watson DI. A case report of Candida albicans costochondritis after a complicated esophagectomy. Plast Reconstr Surg Glob Open 2016;4(1):e608

10 Mohamed AA, Lu XL, Mounmin FA. Diagnosis and treatment of esophageal candidiasis: current updates. Can J Gastroenterol Hepatol 2019;2019:3585136 